\title{
Apresentação Atípica de Leucemia Linfoide Aguda em Criança: Relato de Caso
}

\author{
doi: https://doi.org/10.32635/2176-9745.RBC.2019v65n4.190
}

\author{
Atypical Presentation of Acute Lymphoid Leukemia in Child: Case Report \\ Presettación Atípica de Leucemia Linfoide Aguda en Niño: Relato de Caso
}

Paula Basso Dias; Ana Paula Lopes Luiz²; Leniza Costa Lima Lichtvan³; Adriano Luis Garcia de Lima ${ }^{4}$

Resumo

Introduçáo: A leucemia linfoblástica aguda (LLA) é a neoplasia maligna mais comum em crianças e a principal causa de morte por câncer nessa faixa etária. A hipercalcemia associada a lesões osteolíticas francas é uma rara apresentação da LLA. Relato do Caso: Paciente de 9 anos, sexo masculino, $37 \mathrm{~kg}$, apresentava cefaleia, dor e impotência funcional em membro inferior direito há 15 dias. Exames laboratoriais evidenciaram elevação de velocidade de hemossedimentação e proteína C reativa com hipercalcemia. Nos exames de imagem, apresentava desmineralização óssea e lesões osteolíticas difusas. Aspirado de medula óssea (MO) evidenciou 10\% de blastos, o que não caracterizou leucemia. Pela melhora do quadro clínico, o paciente seguiu com investigação diagnóstica ambulatorialmente. Biópsia de lesão lítica em quadril e novo aspirado de MO detectaram maior número de blastos e confirmaram o diagnóstico de LLA. Iniciou tratamento com protocolo do Grupo Brasileiro de Tratamento de Leucemias na Infância, 2009. Após dois anos, estava bem e sem doença. Conclusáo: A hipercalcemia está associada a apenas $0,6 \%$ a 4,8\% dos casos de LLA. O paciente em questáo apresentava apenas dores ósseas difusas e hipercalcemia, sem a sintomatologia habitual, o que torna seu quadro clínico ainda mais raro, sendo tal apresentação muito escassa na literatura. Apesar de incomuns, hipercalcemia e lesôes osteolíticas difusas podem ser as primeiras e únicas manifestaçóes de LLA na faixa pediátrica. O presente relato torna-se importante ao auxiliar a formulaçáo de diagnósticos precoces da leucemia infantil, mesmo na vigência de um quadro clínico atípico.

Palavras-chave: Leucemia-Linfoma Linfoblástico de Células Precursoras; Hipercalcemia; Diagnóstico Precoce; Criança.

\begin{abstract}
Introduction: Acute lymphoblastic leukemia (ALL) is the most common malignant neoplasm in children and the leading cause of cancer death in this age group. Hypercalcemia associated with frank osteolytic lesions is a rare presentation of ALL. Case Report: 9-year-old male, $37 \mathrm{~kg}$, presented with headache, pain and functional impotence in the lower right leg for 15 days. Laboratory tests showed elevation of erythrocyte sedimentation rate and C-reactive protein with hypercalcemia. Imaging studies revealed bone demineralization and diffuse osteolytic lesions. Bone marrow aspiration showed $10 \%$ of blasts, which did not characterize leukemia. Due to the improvement of his clinical condition, the patient continued the clinical investigation as an outpatient. Biopsy of lytic lesion in the hip and new bone marrow aspirations detected higher number of blasts and confirmed the diagnosis of ALL. Treatment was initiated, following the protocol of the Brazilian Group of Treatment of Leukemia in Childhood, 2009. After two years, he was well and without disease. Conclusion: Hypercalcemia is associated with only $0.6 \%$ to $4.8 \%$ of all ALL cases. The patient presented only diffuse bone pain and hypercalcemia, without the usual symptoms, which makes his clinical condition even rarer, with such presentation being very scarce in the literature. Although uncommon, hypercalcemia and diffuse osteolytic lesions may be the first and only manifestations of ALL in the pediatric range. The present report is important in helping to formulate early diagnosis of childhood leukemia, even in the presence of an atypical clinical condition.

Key words: Precursor Cell Lymphoblastic Leukemia-Lymphoma; Hypercalcemia; Early Diagnosis; Child.
\end{abstract}

Resumen

Introducción: La leucemia linfoblástica aguda (LLA) es la neoplasia maligna más común en los nińos y principal causa de muerte por cáncer en ese grupo de edad. La hipercalcemia asociada a lesiones osteolíticas francas es una rara presentación de LLA. Relato del Caso: Paciente de 9 años, masculino, $37 \mathrm{~kg}$, presentaba cefalea, dolor e impotencia funcional en miembro inferior derecho hace 15 días. Los exámenes de laboratorio evidenciaron elevación de velocidad de sedimentación globular y proteína $C$ reactiva con hipercalcemia. En los exámenes de imagen, presentaba desmineralización ósea y lesiones osteolíticas difusas. Aspirado de médula ósea (MO) evidenció el 10\% de blastos, lo que no caracterizó la leucemia. Debido a la mejora del cuadro clínico, el paciente siguió la investigación diagnóstica ambulatoriamente. La biopsia de lesión lítica en cadera y nuevo aspirado de MO detectaron mayor número de blastos y confirmaron el diagnóstico de LLA. Se inició tratamiento con protocolo del Grupo Brasileńo de Tratamiento de Leucemias en la Infancia, 2009. Conclusión: La hipercalcemia está asociada a sólo 0,6\% a 4,8\% de los casos de LLA. El paciente en cuestión presentaba sólo dolores óseos difusas e hipercalcemia, sin la sintomatología habitual, lo que hace el cuadro clínico del paciente aún más raro, siendo tal presentación muy escasa en la literatura. A pesar de inusual, hipercalcemia y lesiones osteolíticas difusas pueden ser las primeras y únicas manifestaciones de LLA en nińos. El presente relato se vuelve importante al ayudar a la formulación de diagnósticos precoces de la leucemia infantil, incluso en la vigencia de un cuadro clínico atípico.

Palabras clave: Leucemia-Linfoma Linfoblástico de Células Precursoras; Hipercalcemia; Diagnóstico Precoz; Niño.

\footnotetext{
${ }_{1}^{1}$ Faculdade Evangélica do Paraná. Curitiba (PR), Brasil. Orcid iD: https://orcid.org/0000-0002-3163-8854

${ }^{2}$ Faculdade Evangélica do Paraná. Curitiba (PR), Brasil. Orcid iD: https://orcid.org/0000-0002-1767-8171

${ }^{3}$ Hospital Erasto Gaertner (HEG). Curitiba (PR), Brasil. Orcid iD: https://orcid.org/0000-0003-2504-9903

${ }^{4}$ HEG. Curitiba (PR), Brasil. Orcid iD: https://orcid.org/0000-0002-1288-8890

Endereço para correspondência: Adriano Luis Garcia de Lima. Rua Dr. Ovande do Amaral, 201 - Jardim das Américas. Curitiba (PR), Brasil. CEP 81520-060. E-mail: alljekyll@gmail.com
} 


\section{INTRODUÇÃO}

As leucemias são um grupo heterogêneo de neoplasias originadas a partir de células neoplásicas do sistema hematopoiético, em razão da mutação somática e da replicação monoclonal de células progenitoras, que podem ser de linhagem linfoide ou mieloide. Caracterizam-se por infiltração da medula óssea (MO), sangue e outros tecidos. Sua evolução pode levar rapidamente à morte ou progredir de forma mais lenta, sendo dividida em formas agudas ou crônicas, respectivamente ${ }^{1,2}$.

As leucemias linfoblásticas agudas (LLA) são compostas por células $\mathrm{B}$ ou $\mathrm{T}$ imaturas, as quais sáo chamadas de linfoblastos ${ }^{1}$. É o tipo de neoplasia maligna mais comum em crianças e a principal causa de morte por câncer nessa faixa etária ${ }^{3}$. As manifestaçôes clínicas das leucemias linfoblásticas, como anemia, febre e hemorragias, são relacionadas à supressão funcional da MO. São comuns também a presença de dor óssea, linfadenopatia generalizada, esplenomegalia e hepatomegalia. Por outro lado, entre alguns dos sintomas mais raros, vale ressaltar a elevação do cálcio sérico ${ }^{1,2}$.

A hipercalcemia é um achado relativamente comum nas neoplasias malignas que acometem adultos, especialmente naquelas derivadas do sistema hematopoiético, com incidência aproximada em $20 \%$. No entanto, a hipercalcemia associada à malignidade é um distúrbio raro na faixa pediátrica ${ }^{6}$. A hipercalcemia associada a lesóes osteolíticas francas é uma rara complicação da LLA, e apenas alguns poucos casos com essa apresentação foram relatados ${ }^{7}$.

Pela raridade dessa apresentação clínica, este relato de caso tem como objetivo descrever um caso de LLA com sintomatologia não usual, porém possível de ser encontrada, cujo reconhecimento é importante para elaboração do diagnóstico em questão.

\section{RELATO DO CASO}

Paciente de 9 anos, sexo masculino, $37 \mathrm{~kg}$, natural de Paranavaí - PR, sem antecedentes pessoais relevantes, chegou ao Hospital Erasto Gaertner (HEG) em Curitiba - PR, em agosto de 2014, apresentando dor e impotência funcional em membro inferior direito, cefaleia persistente e emagrecimento de 2 quilos ( $5 \%$ do peso total) em um período de 15 dias. No início do quadro, havia realizado exames laboratoriais que evidenciaram elevação de velocidade de hemossedimentação e proteína $\mathrm{C}$ reativa, sem alteraçóes no hemograma. Seus exames de imagem apontavam desmineralização óssea de quadril.

$\mathrm{Na}$ primeira avaliação, apresentava-se em bom estado geral, restrito ao leito, hidratado, hipocorado, eupneico, afebril e com dor à abdução de perna direita. Não apresentava quaisquer outras alteraçóes.

Nos exames laboratoriais de entrada realizados no HEG, evidenciou-se uma hipercalcemia importante $(\mathrm{Ca}$ total $15,4 \mathrm{mg} / \mathrm{dL}$; valor de referência: 8,5 a $11,0 \mathrm{mg} /$ dl). Foram iniciadas medidas para redução do cálcio sérico, associadas a diurético e hidratação. Paciente foi encaminhado para UTI já no primeiro dia de internamento para monitorização contínua. Diante de tal quadro, foi submetido a novos exames, tanto laboratoriais quanto de imagem.

Nas radiografias e tomografias realizadas dois dias após a admissão, o paciente apresentava desmineralização óssea com lesôes líticas difusas em crânio, coluna vertebral, quadril, membros inferiores e escápulas (Figuras 1 e 2). Ultrassonografias cervical, de tireoide e abdômen total estavam normais.

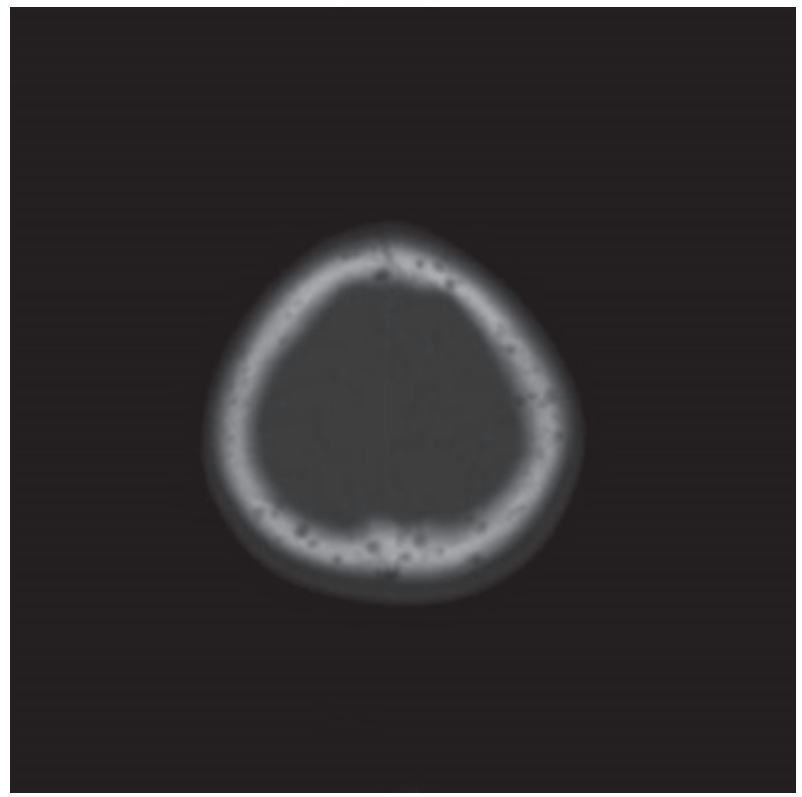

Figura 1. Tomografia axial computadorizada evidenciando lesões osteolíticas no crânio

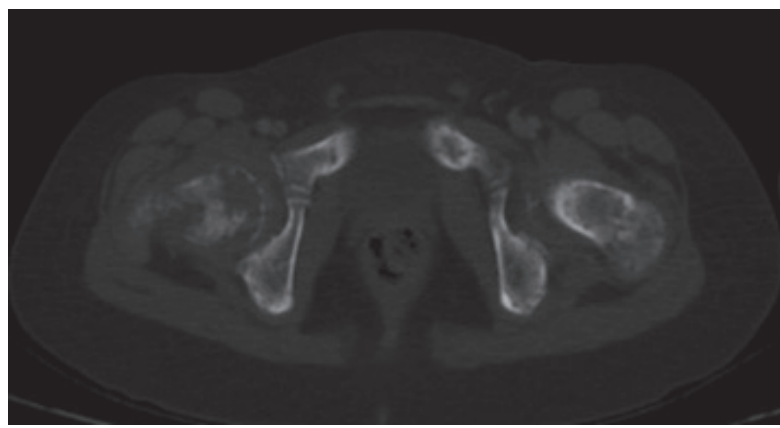

Figura 2. Tomografia axial computadorizada evidenciando lesões osteolíticas no quadril

Entre as hipóteses diagnósticas, foram consideradas hiperparatireoidismo (sendo adenoma a causa mais 
comum) e leucemia. Foram realizadas dosagem de paratormônio (PTH) sérico e cintilografia com Tecnécio-99m SestaMIBI (radiofármaco que se concentra nas células oxifílicas da paratireoide, consideradas hiperfuncionantes - adenoma ou hiperplasia - as áreas focais que mantêm captação persistente tardiamente, aproximadamente duas horas após aplicação) ${ }^{8}$ ambas normais, sendo descartada a hipótese de hiperparatireoidismo no sétimo dia de internamento. Aspirado de MO evidenciou apenas 10\% de células imaturas, o que não caracteriza leucemia, já que são necessários mais de $20 \%$ de blastos em medula para confirmação diagnóstica?

Em virtude da boa redução do cálcio sérico e da melhora das dores, o paciente recebeu alta oito dias após a admissão com analgesia simples, e seguiuse investigação diagnóstica ambulatorialmente. Repetiram-se exames laboratoriais e as novas dosagens de cálcio e PTH foram normais, bem como o hemograma, cinco dias após a alta.

Como o diagnóstico ainda permanecia inconclusivo, foi realizada biópsia de lesão lítica em região de quadril e novo aspirado de $\mathrm{MO}$ em setembro de 2014, uma semana após a alta. Novos exames anatomopatológicos e de imunofenotipagem detectaram a presença de maior número de blastos em $\mathrm{MO}$ e confirmaram o diagnóstico final de LLA. Foi iniciado tratamento com protocolo do Grupo Brasileiro de Tratamento de Leucemias na Infância $\left(\right.$ GBTLI-LLA) ${ }^{10}$.

Paciente evoluiu gradativamente e, em menos de duas semanas do início do tratamento, já se apresentava completamente sem dor e caminhava com apoio. Teve ótima resposta e, após quase dois anos da admissão no hospital, em abril de 2016, estava bem e com ausência de doença.

Em sua última consulta de rotina para reavaliaçáo, em abril de 2017, foi identificada recidiva da doença isolada em testículo esquerdo. Reiniciou tratamento quimioterápico com novo protocolo. Apresenta-se sem novas queixas até o presente momento.

O presente estudo foi aprovado pelo Comitê de Ética em Pesquisa (CEP) da Liga Paranaense de Combate ao Câncer sob o no. CAAE: 91839118.3.0000.0098. A autorização para publicação foi fornecida pelos responsáveis da criança, por meio de assinatura do Termo de Assentimento Livre e Esclarecido.

\section{DISCUSSÃO}

As LLA do tipo B (LLA-B) correspondem a aproximadamente $85 \%$ das LLA, e apresentam sinais e sintomas relacionados à supressão da hematopoiese normal, causada pelo acúmulo de células tumorais na $\mathrm{MO}^{2}$. Assim, os sintomas mais comuns das LLA como fadiga, febre e hemorragia estáo relacionados com a depressão funcional da medula. Outros sintomas, como dor óssea, linfadenopatia generalizada, esplenomegalia, hepatomegalia e dilatação testicular são efeitos em massa causados pela infiltração neoplásica. Podem ocorrer também manifestaçóes do sistema nervoso central, como cefaleia, vômito e paralisia de nervos, resultantes de expansão meníngea. Entre o quadro de sinais e sintomas que podem ser encontrados, uma manifestação rara das LLA é a hipercalcemia ${ }^{2}$. O paciente do presente relato apresentava apenas dores ósseas difusas e hipercalcemia, sem evidências da sintomatologia mais habitual.

Hipercalcemia é um achado que pode aparecer em neoplasias malignas em adultos, em 5\% a 20\% dos casos, mesmo na ausência de metástases ósseas ${ }^{11}$. Já na faixa etária pediátrica, é uma manifestação muito mais incomum das neoplasias malignas, associada a apenas $0,4 \%$ a $1,3 \%$ dos casos $^{6}$. A incidência de hipercalcemia em crianças com leucemia fica em torno de $0,6 \%$ a $4,8 \%{ }^{12}$. São vários os possíveis mecanismos associados à hipercalcemia, com destaque para destruição óssea localizada por infiltração de células cancerosas, atividade osteoclástica alterada por causa da influência de fatores secretados pelas células cancerosas, com produção aumentada de vitamina $\mathrm{D}$, $\mathrm{PTH}$ ou proteína relacionada ao $\mathrm{PTH}^{2}$. Estudo também sugere que um aumento nos níveis de certas citocinas, como IL-1, IL-6, TNF, PGE2 e TGF-alfa, pode ser responsável pela hipercalcemia em LLA ${ }^{13}$. Acredita-se que as citocinas atuam como fatores ativadores de osteoclastos, resultando em lesóes osteolíticas e osteopenia, principalmente no esqueleto axial ${ }^{14}$. Um estudo realizado no Hospital St Jude, nos EUA, identificou hipercalcemia em dez de 2.816 crianças que apresentavam LLA, mas em apenas sete delas a hipercalcemia estava presente no início do quadro ${ }^{11}$. Outro estudo, realizado no Japão, identificou, em um período de 15 anos, um total de 25 crianças com LLA associada à hipercalcemia. Cerca de 7.500 crianças foram diagnosticadas com LLA durante esse período ${ }^{15}$. Esses artigos corroboram a raridade do sintoma manifestado pelo paciente relatado.

A terapêutica da hipercalcemia inclui hiper-hidratação, bifosfonatos e tratamento da doença de base. Na pediatria, bifosfonatos ainda não são muito utilizados em razáo da relação com efeitos adversos à osteogênese ${ }^{6}$. Porém, inicialmente foram as medidas instituídas no paciente descrito.

Outra manifestação também incomum da LLA é a presença de lesôes osteolíticas não associadas com infiltração neoplásica. O quadro torna-se ainda mais raro quando a presença de tais lesôes e hipercalcemia estâo presentes em um mesmo paciente, sendo tal apresentação muito escassa na literatura ${ }^{16}$. 
Em relação aos achados encontrados no caso em discussão, a presença de hipercalcemia em criança, contagem normal de linfócitos, ausência de blastos no sangue periférico, ausência de linfadenopatias, presença de lesôes osteolíticas e ausência de hepatomegalia e esplenomegalia foram descritas em apenas outros dois casos na literatura mundial7,17. Entretanto, mais de 20 casos já foram relatados com apresentação clínica semelhante, que relacionam a presença de hipercalcemia e de LLA em crianças. Outros casos descritos mostram a presença de hipercalcemia e lesões osteolíticas em pacientes pediátricos, mas descrevem linfadenopatias ${ }^{18,13} \mathrm{e}$ hepatoesplenomegalia ${ }^{13}$ associadas. Na literatura brasileira, nenhum outro caso como o apresentado neste artigo foi encontrado.

Há controvérsias sobre a mudança do prognóstico relacionado com a presença de lesões osteolíticas e hipercalcemia em pacientes com LLA. Alguns estudos sugerem que não existe relação entre a presença das lesôes e o pior prognóstico de pacientes com LLA ${ }^{9,12,19,20}$. Há evidência na literatura de que o período de remissão da doença é reduzido em pacientes com lesóes osteolíticas ${ }^{11}$.

\section{CONCLUSÃO}

Ainda que sejam achados incomuns, a hipercalcemia e as lesôes osteolíticas difusas podem ser as primeiras manifestaçóes da LLA na faixa pediátrica. Portanto, o presente relato torna-se importante ao auxiliar a formulaçâoo de diagnósticos precoces da leucemia infantil, mesmo na vigência de um quadro clínico atípico.

\section{CONTRIBUIÇÕES}

Os autores contribuíram igualmente de forma substancial em todas as etapas do trabalho e aprovaram a versão final a ser publicada.

\section{DECLARAÇÃO DE CONFLITO DE INTERESSES}

Nada a declarar.

\section{FONTES DE FINANCIAMENTO}

Não há.

\section{REFERÊNCIAS}

1. Sánchez MAO, Ortega MLO, Barrientos JVR. Leucemia linfoblástica aguda. Med Int Mex. 2007;23(1):26-33.

2. Kumar V, Abbas AK, Fausto N, et al. Robbins e Cotran patologia: bases patológicas das doenças. 7. ed. Rio de Janeiro: Elsevier; 2005.
3. Pedrosa F, Lins M. Leucemia linfoide aguda: uma doença curável. Rev Bras Saúde Mater Infant. 2002;2(1):63-68. doi: http://dx.doi.org/10.1590/S151938292002000100010

4. Mundy GR, Ibbotson KJ, D'Souza SM, et al. The hypercalcemia of cancer: clinical implications and pathogenic mechanisms. N Engl J Med 1984;310(26):1718-27. doi: http://dx.doi.org/10.1056/ NEJM198406283102607

5. Oloomi Z. Acute lymphoblastic leukemia without circulating blasts presenting as severe hypercalcemia. Acta Medica Iranica. 2007;45(1):76-8.

6. Martins AL, Moniz M, Nunes PS, et al. Severe hypercalcemia as a form of acute lymphoblastic leukemia presentation in children. Rev Bras Tera Intensiva. 2015;27(4):402-5. doi: http://dx.doi.org/10.5935/0103507X.20150067

7. Soni PN. Hypercalcaemia and multiple osteolytic lesions in childhood acute lymphoblastic leukaemia. Postgrad Med J. 1993;69(812):483-5. doi: http://dx.doi. org/10.1136/pgmj.69.812.483

8. Greenspan BS, Dillehay G, Intenzo C, et al. SNM practice guideline for parathyroid scintigraphy 4.0. J Nucl Med Technol. 2012 Jun;40(2):111-118.

9. Cavalcante MS, Santana ISR, Torres F. Leucemia linfoide aguda e seus principais conceitos. Rev Cient FAEMA. 2017 Dez;8(2):151-64. doi: https://doi.org/10.31072/ rcf.v8i2.578

10. Sociedade Brasileira de Oncologia Pediátrica. Protocolo brasileiro de tratamento da leucemia linfóide aguda na infância GBTLI LLA-2009. São Paulo: Campinas; 2011.

11. McKayC, Furman WL. Hypercalcemiacomplicatingchildhood malignancies. Cancer. 1993;72(1):256-60. doi: https://doi. org/10.1002/1097-0142(19930701)72:1<256::AIDCNCR2820720145>3.0.CO;2-D

12. Trehan A, Cheetham T, Bailey S. Hypercalcemia in acute lymphoblastic leukemia: an overview. J Pediatr Hematol Ocol. 2009;31(6):424-7. doi: https://doi.org/10.1097/ MPH.0b013e3181a1c12b

13. Peterson K, Higgins R, Peterson T, et al. Osteolytic bone lesions, hypercalcemia, and renal failure: a rare presentation of childhood acute lymphoblastic leukemia. Am J Cancer Case Rep. 2013;1(2).

14. Mahmood K, Ubaid M, Taliya Rizvi S. Multiple osteolytic lesions causing hypercalcemia: a rare presentation of acute lymphoblastic leukemia. Case Rep Med. 2017;2017:2347810. doi: https://doi. org/10.1155/2017/2347810

15. Inukai $T$, Hirose $K$, Inaba $T$, et al. Hypercalcemia in childhood acute lymphoblastic leukemia: frequent implication of parathyroid hormone-related peptide and E2A-HLF from translocation 17;19. Leukemia. 2007;21(2):288-96. doi: https://doi.org/10.1038/ sj.leu. 2404496 
16. Bhat GM. A child with acute lymphoblastic leukemia (ALL) presenting with symptomatic hypercalcemia and multiple osteolytic lesions. Indian J Med Paediatr Oncol. 2007;28(3):46-7.

17. Dhivyasree S, Dhivyalakshmi J, Sankaranarayanan S, et al. Severe hypercalcemia: a rare and unusual presentation of acute lymphoblastic leukemia. J Cancer Res Ther. 2018;14(12):1244-46. doi: 10.4103/0973-1482.187240

18. Bechir A, Haifa R, Atef BA, et al. Osteolytic bone lesions, severe hypercalcemia without circulating blasts: unusual presentation of childhood acute lymphoblastic leukemia. Pan Afr Med J. 2017 Apr 28;26:244. doi: https://doi. org/10.11604/pamj.2017.26.244.10506

19. Pui CH, editor. Childhood leukemias. Cambridge, UK: Cambridge University Press; 2000. Chapter 29, Acute complications; p. 443-462

20. Lokadasan R, Prem S, Koshy SM, et al. Hypercalcaemia with disseminated osteolytic lesions: a rare presentation of childhood acute lymphoblastic leukaemia. Ecancermedicalscience. 2015;9:542. doi: https://doi. org/10.3332/ecancer.2015.542 